\title{
The epidemiology of premature aging and associated comorbidities
}

This article was published in the following Dove Press journal:

Clinical Interventions in Aging

2 August 2013

Number of times this article has been viewed

\section{Fabio Coppedè}

Department of Translational Research and New Technologies in Medicine and Surgery, University of Pisa, Pisa, Italy
Correspondence: Fabio Coppedè Department of Translational Research and New Technologies in Medicine and Surgery, University of Pisa,

Via S Giuseppe 22, 56I 26 Pisa, Italy

Tel +3905022I 1028

Fax +39050 22I 1034

Email f.coppede@geog.unipi.it
Abstract: Hutchinson-Gilford Progeria Syndrome and Werner syndrome, also known as childhood- and adulthood-progeria, respectively, represent two of the best characterized human progeroid diseases with clinical features mimicking physiological aging at an early age. The discovery of their genetic basis has led to the identification of several gene mutations leading to a spectrum of progeroid phenotypes ranging from moderate and mild-severe to very aggressive forms. In parallel, the creation of disease registers and databases provided available data for the design of relatively large-scale epidemiological studies, thereby allowing a better understanding of the nature and frequency of the premature aging-associated signs and symptoms. The aim of this article is to review the most recent findings concerning the epidemiology of premature aging disorders, their genetic basis, and the most recent reports on the frequency of associated diseases.

Keywords: Hutchinson-Gilford Progeria Syndrome, Werner syndrome, premature aging disorders, epidemiology, cardiovascular diseases, cancer, atherosclerosis, genetics, sign and symptoms

\section{Introduction}

Hutchinson-Gilford Progeria Syndrome (HGPS) and Werner syndrome (WS) are two of the best characterized human progeroid diseases with clinical features mimicking physiological aging at an early age, the first being referred as to childhood progeria, and the latter as progeria of adulthood. ${ }^{1}$ An increasing number of intermediate progeroid phenotypes, known as atypical progeroid syndromes, atypical HGPS, or atypical WS, have been described. ${ }^{1}$ The aim of this review article is to describe the most recent findings concerning the epidemiology of premature aging disorders, their genetic basis, and the most recent reports on the frequency of associated diseases.

\section{Hutchinson-Gilford Progeria Syndrome}

HGPS is an extremely rare genetic disorder affecting about one per four to eight million live births. ${ }^{2}$ More precisely, the reported prevalence rate of the disease is one in eight million births, but if unreported or misdiagnosed cases are taken into account, the estimated birth prevalence is one in four million. ${ }^{2}$

According to the Progeria Research Foundation database (http://www. progeriaresearch.org/prf-by-the-numbersprf.html), there are an estimated 200-250 children living with progeria worldwide at any one time, and 103 of them have been identified as of April 2013. Progeria affects both sexes and all races, and HGPS cases have been discovered in over 40 different countries. In particular, a map 
of ascertained HGPS children is available at the Progeria Research Foundation portal (http://www.findtheother150. org/), and there are records of 20 cases in Northern America, 16 cases in Central and Southern America, 24 cases in Europe and the Mediterranean regions, four cases in Africa, and 18 cases in Asia.

The disease was named after the reports by Jonathan Hutchinson and Hastings Gilford, the doctors who first described it in England, and it is classified as a segmental progeroid syndrome since multiple organs and tissues replicate phenotypes associated with normal aging. ${ }^{3,4}$

Children with HGPS appear healthy at birth but develop distinctive clinical features during the first years of their life, including severe growth retardation, usually associated with skeletal alteration as well as loss of subcutaneous fat and skin appendages, and some developmental processes are delayed (dentition) or absent. ${ }^{4}$ Death occurs in those affected by their early teenage years, and usually results from heart attacks and strokes. ${ }^{1}$

The majority of classical HGPS is caused by a de novo point mutation in exon 11 of the $L M N A$ gene (c. $1824 \mathrm{C}>\mathrm{T}$, p.G608G). ${ }^{5}$ The $L M N A$ gene encodes A-type lamins, which are intermediate filament proteins of the inner nuclear lamina. The c. $1824 \mathrm{C}>\mathrm{T}$ mutation results in the activation of a cryptic splice donor site that removes 150 nucleotides from exon 11 . The resulting lamin $\mathrm{A} \Delta 150$ messenger ribonucleic acid gives rise to a lamin $\mathrm{A}$ isoform containing an internal deletion of 50 amino acids, known as progerin (a protein that cannot undergo complete maturation). ${ }^{5}$ Lamins constitute the major component of the nuclear lamina; in addition to providing structure and shape to the nucleus, they are involved in organizing several processes including chromatin organization, deoxyribonucleic acid (DNA) replication, transcription, DNA methylation and epigenetic regulation, and DNA repair. ${ }^{6}$ Those mechanisms are impaired in HGPS and might contribute to the progeria phenotype. Indeed, cells obtained from HGPS patients show a markedly reduced lifespan when grown in culture, and accumulate defects in nuclear structure and architecture with cell passaging, including lobulation of the nuclear envelope, thickening of the nuclear lamina, loss of peripheral heterochromatin, and clustering of nuclear pores, which are accompanied by an increase in the amount of progerin within the cells. ${ }^{7}$

\section{HGPS-associated symptoms}

A visible vein across the nasal bridge is often the first observable sign in HGPS infants. A profound failure to thrive occurs during the first year, usually from 6 months to 12 months.
On average, HGPS children gain $0.4-0.5 \mathrm{~kg} /$ year and reach a final weight of about $14-15 \mathrm{~kg}$ and a final height of $110 \mathrm{~cm}$. Progressive alopecia usually takes place within 6 months to 2 years, and between the ages of 2 years and 3 years, most children become bald. ${ }^{8}$ Other symptoms become apparent during the first year to third year, including characteristic facies, loss of subcutaneous fat, stiffness of joints, bone changes, and abnormal tightness of the skin over the abdomen and upper thighs. ${ }^{1}$ With time, the skin becomes thin, dry, and atrophic, with reduced turgor and sometimes with hyperkeratosis. Small, light-brown spots frequently develop on the neck and upper thorax, and subsequently on the scalp and limbs. Typical facial abnormalities include a receding mandible, a small and beaked nose, prominent scalp veins, prominent eyes, and protruding ears that lack lobules. The facial characteristics gradually develop and both the face and body change with time: the subcutaneous fat in the face disappears completely and the facial muscles decrease in size. The body shows increasing loss of subcutaneous fat and muscle bulk and the joints protrude. ${ }^{1,89}$ At the bone level, patients show clavicular hypoplasia, generalized osteopenia, and acroosteolyses of distal phalanges. Motor and mental development is normal, cognitive functions are preserved, and the children follow a normal psychosocial development and show normal behaviors for their age. ${ }^{1,9}$ Dentition is delayed and crowded. ${ }^{9}$ HGPS individuals have a high-pitched voice, do not reproduce, and their appearance becomes like that of an older person with time. ${ }^{1,8}$

Additional findings that are present in some but not all affected individuals include excessive ocular tearing, photophobia, exposure keratitis, and Raynaud's phenomenon. ${ }^{9}$ Most children die in their early teens from heart attacks and strokes caused by progressive atherosclerotic disease, with myocardial infarction representing the most frequent cause of death at a mean age of around 13 years. ${ }^{8}$ A comprehensive description of most of the HGPS-associated manifestations is provided in the following sections.

\section{Cardiovascular disease in HGPS}

Cardiovascular disease (CVD) represents the principal factor affecting mortality in HGPS individuals, with death resulting from myocardial infarction, stroke, or congestive cardiac failure in $75 \%$ of cases. ${ }^{10}$ Cardiovascular problems are absent during the first 5 years of life, but children gradually develop shortness of breath with exertion and easy fatigability from the age of 6-8 years. ${ }^{10}$ Accelerated CVD leads to debilitating morbidity in HGPS and culminates in mortality from myocardial infarction or stroke at an average age of 13 years. ${ }^{11}$ 
The rapid progression of CVD in HGPS presents an opportunity to explore the natural history of human CVD, and a study performed on 26 HGPS patients and 22 matched controls revealed that the carotid-femoral pulse wave velocity was dramatically elevated in patients. ${ }^{11}$ Carotid duplex ultrasound echobrightness, assessed as a measure of arterial wall density, was significantly greater than age- and sexmatched controls in the intima-media, near adventitia, and deep adventitia, as was internal carotid artery mean flow velocity. ${ }^{11}$ Overall, those data demonstrated that, along with peripheral vascular occlusive disease, accelerated vascular stiffening is an early and pervasive mechanism of vascular disease in HGPS. ${ }^{11}$

Autopsy data have shown widespread atherosclerosis in HGPS patients. Particularly, advanced coronary atherosclerotic lesions have been reported, and the arteries were frequently stenosed or occluded by plaques or narrowing of intramural arteries. Occlusion of the right coronary artery, lesions of the left anterior descending artery, and severe atherosclerosis of the aorta, represent common findings in HGPS. ${ }^{10}$ Valvular changes and pulmonary arterial lesions have also been reported in HGPS individuals. ${ }^{10}$

\section{Cerebrovascular disease in HGPS}

Cerebrovascular arteriopathy and stroke have been recently assessed by means of a neurovascular imaging cohort study of HGPS, a study aimed to identify the neurovascular features, infarct type, topography, and natural history of stroke. ${ }^{12}$ A total of 25 children with confirmed diagnoses of HGPS were included in the study, which revealed a vasculopathy unique to HGPS, including distinctive intracranial stenoocclusive arterial lesions, basal cistern collateral vessels, and slow compensatory collateral flow over the cerebral convexities. Moreover, the authors identified early and clinically silent strokes as a prevalent disease characteristic in HGPS. Indeed, a radiographic evidence of infarction was found in $60 \%$ of patients, of which half were likely clinically silent. ${ }^{12}$

\section{Skeletal abnormalities in HGPS}

A recent study performed with an inducible and tissue-specific mouse model, which expresses the most common HGPS mutation (c.1824C $>$ T) in osteoblasts and odontoblasts, revealed that the expression of the HGPS mutation during osteoblast development results in a loss of osteocytes, irregular mineralization, and poor biomechanical properties. ${ }^{13}$

A comprehensive survey of the skeletal dysmorphisms observed in children with HGPS using conventional radiography was obtained from 39 children with the classic HGPS genotype, representing approximately 15\%-20\% of the world's HGPS population. ${ }^{14}$ Small clavicles were observed in $100 \%$ of the patients; followed by coxa valga and acroosteolysis, which were observed in more than $90 \%$ of the patients; and resorption of the distal clavicles and narrow apices, both present in $82 \%$ of the subjects. Other frequent skeletal abnormalities were hip dysplasia (69\%) and thin ribs (59\%). In addition, $30 \%$ to $45 \%$ of the patients showed resorption of the anterior ribs, closed sagittal suture, generalized osteopenia, focal cortical defects, flexed fingers, ulnar minus variant, or enlarged heart. Less frequent (20\%-30\%) were dystrophic calcification, sagittal suture diastasis, enlarged femoral head, pseudoarthrosis, enlarged femoral greater trochanter, and avascular necrosis of the proximal femur. Kyphoscoliosis, enlarged humoral head, narrowed humoral diaphysis, prominent pulmonary vessels, and wormian bones were reported in less than $15 \%$ of the patients, and other abnormalities, such as accentuated osteopenia of proximal humoral/femoral epiphysis, rib fracture, Madelung deformity, ivory epiphyses, bifid rib, and congenitally fused ribs were reported in $10 \%$ or less of the patients. ${ }^{14}$

\section{Craniofacial abnormalities in HGPS}

Using the Progeria Research Foundation medical and research database (http://www.progeriaresearch.org/ medical database.html), data on 25 HGPS patients have been examined in order to provide an overview of the craniofacial abnormalities in progeria. ${ }^{15}$

Concerning scalp, calvarial, and skull base features, thinning of the calvarium was seen in $95 \%$ of the individuals, often accompanied by a paucity of scalp fat (91\%). A mottled appearance of the skull was seen in $59 \%$ of the patients. Two individuals ( $8 \%$ ) had skull fractures, and prominent vascular markings of the bony calvaria were observed in $90 \%$ of the subjects. Craniofacial disproportion (a large cranium relative to the facial size), and a J-shaped sella were observed in almost $90 \%$ of the patients, and a delayed closure of the anterior fontanel was seen in $56 \%$ of them. ${ }^{15}$

Concerning oral maxillary, zygomatic arch, and parotid gland features, the authors observed a short mandibular ramus in $83 \%$ of the patients, with a gracile thin zygomatic arch in $50 \%$ of them. A shallow glenoid fossa with a hypoplastic or absent articular eminence and flattening of the mandibular condyle were seen in $43 \%$ of the patients. Moreover, $45 \%$ of the children had a V-shaped palate, and $50 \%$ of them had disorganized dentition. A prominent parotid gland was seen in all the children analyzed. ${ }^{15}$ 
With regard to orbital features, hypotelorism was noted in $86 \%$ of the children, and kinking of the optic nerves was seen in $89 \%{ }^{15}$

\section{Other manifestations in HGPS}

Fifteen patients with HGPS have been enrolled in a prospective study to evaluate otologic and audiologic manifestations. ${ }^{16}$ All patients had stiff auricular cartilages, small or absent lobules, and hypoplasia of the lateral softtissue portion of the external ear canal leading to a shortened canal. A low-frequency conductive hearing loss in the $250 \mathrm{~Hz}$ to $500 \mathrm{~Hz}$ range was observed in $86.4 \%$ of the ears, despite largely normal tympanometry. ${ }^{16}$ In addition, $71 \%$ of the patients had dry cerumen impaction, and $29 \%$ of them reported a history of recurrent otitis media. ${ }^{16}$

Insulin resistance occurs in about $50 \%$ of affected patients without progression to diabetes mellitus. ${ }^{9}$ A rare case of a 10-year-old boy with genetically confirmed classical HGPS and hypoparathyroidism has been reported. ${ }^{17}$ Differently from other premature aging syndromes, such as WS or others caused by mutations of DNA repair genes, there is no reported increase of cancer incidence in HGPS patients. ${ }^{8,18}$ Cataracts are not frequent. ${ }^{8}$ We recently reviewed neurodegenerative signs or symptoms in premature aging disorders, and they are absent in HGPS individuals. ${ }^{1}$

\section{From mild to severe progeria: the genetic basis of classical HGPS and atypical progeria syndromes}

Although the majority (approximately 90\%) of classical HGPS is caused by a de novo point mutation in exon 11 of the $L M N A$ gene (c.1824C>T, p.G608G), it was clear from the beginning that other mutations in $L M N A$ could cause a similar phenotype. ${ }^{5}$ Particularly, the genetic basis of HGPS was identified in 2003 by two independent research groups. ${ }^{5,19}$ Eriksson et $\mathrm{al}^{5}$ observed the G608G mutation in 90\% of 20 HGPS individuals, and De Sandre-Giovannoli et al ${ }^{19}$ observed it in two affected children. A patient with a c.1822G $>$ A mutation (p.G608S), with classical HGPS, was also recorded by Eriksson et al. ${ }^{5}$

The $L M N A$ gene encodes the four different A-type lamins (lamin A, lamin A $\Delta 10$, lamin C, and lamin C2), which are intermediate filament proteins of the inner nuclear lamina. ${ }^{1}$ Lamin A (encoded by exons 1-12) and lamin C (encoded by exons 1-10) are the major proteins expressed in differentiated cells. Lamin A $\Delta 10$ is identical to lamin A except that it lacks exon 10 and has been detected in cells from colon and placenta, in leukocytes and fibroblasts, and in tumor cells.
Lamin C2 has an alternative exon 1 compared with lamin $\mathrm{C}$ and is present in germ cells. ${ }^{1}$ Lamin A proteins contain CaaX boxes at their C-terminal ends; they are synthesized as prelamin A proteins, which undergo farnesylation and other posttranslational modifications to become mature proteins (Figure 1).

The p.G608G mutation results in the activation of a cryptic splice donor site, leading to the production of a lamin A isoform containing an internal deletion of 50 amino acids, known as progerin - a protein that cannot undergo complete maturation. ${ }^{5}$ Particularly, the deletion eliminates the site for endoproteolitic cleavage by zinc metalloproteinase Ste24 homolog (ZMPSTE24), a cleavage required for the production of mature lamin A. The recurrent c. $1824 \mathrm{C}>\mathrm{T}$ mutation causing HGPS is a de novo dominant point mutation, mostly originating on the paternal allele and often linked with advanced paternal age. ${ }^{5}$

The zinc metalloprotease, ZMPSTE24, plays a critical role in nuclear lamin biology by cleaving the prenylated and carboxylmethylated 15 -amino acid tail from the C-terminus of prelamin A to yield mature lamin A (Figure 1). Two patients with extraordinarily severe forms of progeria caused by unusual mutations in $L M N A$ have been described. ${ }^{20}$ Both mutations (IVS11+1G $>$ A and p.V607V) resulted in a strong activation of the aberrant splice site observed in typical HGPS, leading to increased progerin expression compared to typical HGPS cases. ${ }^{20}$ By contrast, two subjects bearing a missense (p.T623S) mutation leading to the deletion of 35 amino acids in exon 11 of LMNA showed a less aggressive progeroid phenotype (slowly progressing progeria) compared to classical HGPS. ${ }^{21,22}$ Overall, the amount of toxic progerin in cells appears to correlate with the severity of disease outcomes. ${ }^{20}$

Interestingly, recessive mutations in ZMPSTE24 also disrupt lamin A proteolytic processing and are associated with three distinct but related human diseases that share features of premature aging, with a gradation of severity. ${ }^{23}$ Indeed, ZMPSTE24 mutations cause: (1) the mild progeroid disorder mandibuloacral dysplasia, a rare autosomal recessive disorder characterized by postnatal growth retardation, craniofacial anomalies, skeletal malformations, and mottled cutaneous pigmentation; (2) a severe form of progeria denoted as atypical HGPS; and (3) restrictive dermopathy (RD), a fatal neonatal disorder characterized by severe intrauterine growth delay that can be considered as an "extreme form" of premature aging. ${ }^{23}$ Twenty human ZMPSTE24 alleles have been identified that are associated with those diseases. They have been recently reviewed by 


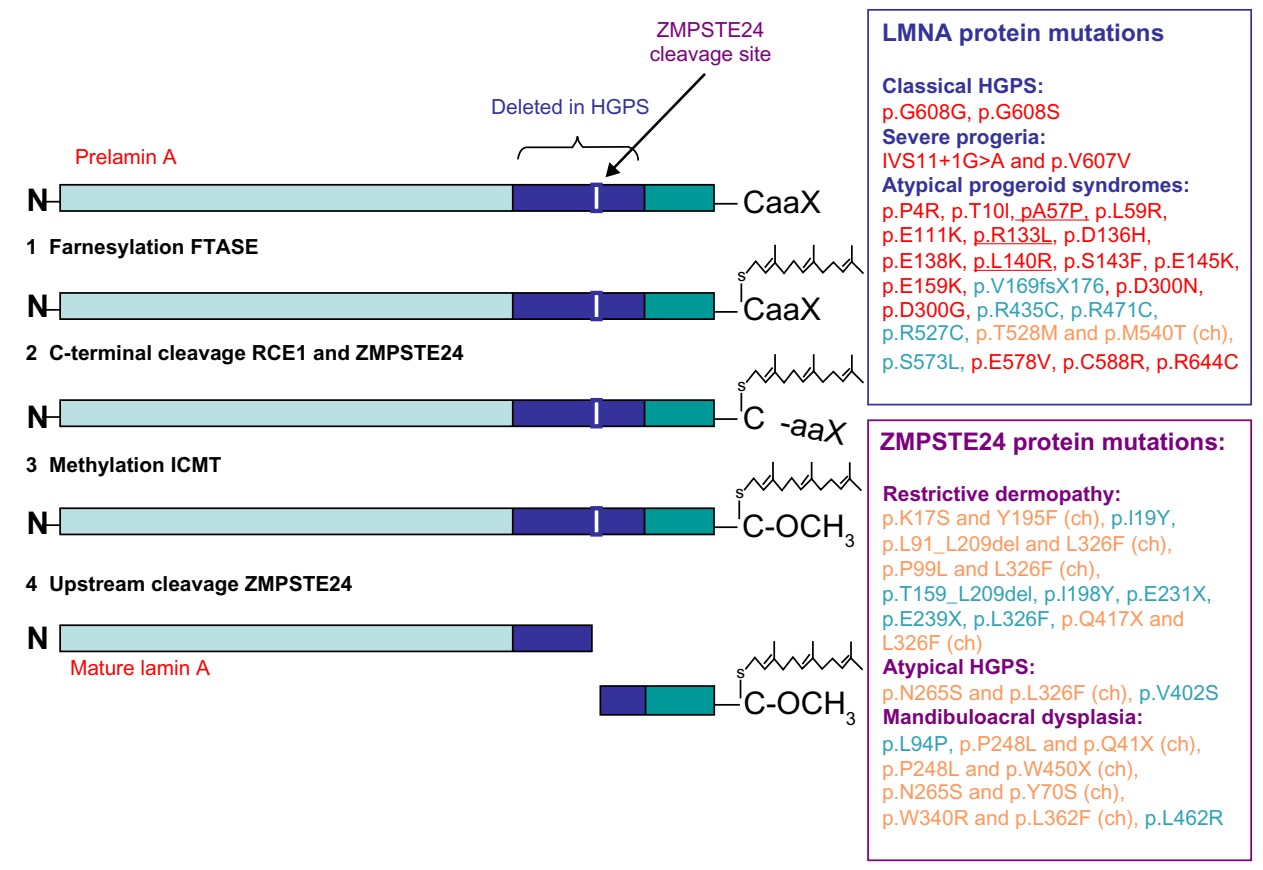

Figure I Representation of prelamin A processing and summary of LMNA and ZMPSTE24 mutations leading to classical and atypical progeria.

Notes: Prelamin A requires maturation to become mature lamin A. First, the C-terminal end of prelamin A contains a CaaX motif, which is modified by farnesylation of the cysteine residue (I), mediated by a FTASE protein, and is followed by the cleavage of the (2) -aaX terminal residues that can be performed either by RCEI or by ZMPSTE24. (3) After the cleavage, the cysteine residue is carboxymethylated by ICMT. The last step removes the C-terminal 15 residues through cleavage by (4) ZMPSTE24, yielding mature lamin A. In HGPS cells bearing the common LMNA p.G608G mutation, the activation of the cryptic splice site results in the deletion of a 50 aa region from prelamin A (indicated in dark blue in the figure), which contains the ZMPSTE24 cleavage site (indicated in the figure). As a result, the P.G608G mutation leads to the production and the accumulation of a smaller prelamin A protein, which cannot undergo complete maturation, termed progerin. A diagnostic test is available for the common p.G608G mutation, as well as for the less common LMNA splicing mutations, namely p.G608S, p.V607V, or IVSII+IG >A, all resulting in the activation of the cryptic splice site. Several other LMNA mutations have been reported in a small number of patients and these lead to a spectrum of progeroid phenotypes ranging from mild-moderate to very aggressive forms, and are referred to as atypical progeroid syndromes. Those mutations can be either dominant (in red ink) or recessive (in green ink) and can alter residues throughout the protein structure with no clear clustering in a single region of lamin A. The term (ch) indicates a compound heterozygous mutation (in orange ink). Underlined LMNA mutations are those observed in atypical WS cases. Recessive ZMPSTE24 mutations also disrupt prelamin A processing and are associated with three distinct, but related, human diseases that share features of premature aging, with a gradation of severity: mandibuloacral dysplasia, atypical HGPS, and restrictive dermopathy. Concerning ZMPSTE24 mutations, the term (ch) indicates a compound heterozygous mutation (in orange ink), otherwise the mutation was found in homozygosis in the patient (in green ink).

Abbreviations: HGPS, Hutchinson-Gilford Progeria Syndrome; ZMPSTE24, zinc metalloprotease related to Ste24p; FTASE, farnesyltransferase; RCEI, Ras-converting enzyme I; ICMT, isoprenylcysteine carboxyl methyltransferase; WS, Werner syndrome.

Barrowman et al ${ }^{24}$ who demonstrated a correlation between decreasing ZMPSTE24 protease activity and increasing disease severity. Particularly, complete loss-of-function alleles are associated with $\mathrm{RD}$, whereas retention of partial, measurable activity results in mandibuloacral dysplasia or severe progeria. ${ }^{23}$ Figure 1 illustrates most of the known LMNA and ZMPSTE24 mutations linked to progeroid diseases.

Although the mechanism whereby persistently farnesylated lamin A, either resulting from LMNA or ZMPSTE24 mutations, causes premature aging phenotypes is unknown, changes in chromatin architecture and in histone methylation and gene expression, defective DNA repair and accumulation of DNA damage, impaired structural and mechanical properties of the nuclear lamina, and perturbations in transcription factors and nuclear proteins, are among the suggested pathologic mechanisms. ${ }^{1,8}$
The diagnosis of HGPS is based on the recognition of common clinical features and the detection of either the c. $1824 \mathrm{C}>\mathrm{T}$ (p.G608G) heterozygous $L M N A$ mutation in the classic form of HGPS, or one of three of the heterozygous LMNA mutations in atypical HGPS: c.1822G $>$ A (p.G608S), c. $1821 \mathrm{G}>\mathrm{A}(\mathrm{p} . \mathrm{V} 607 \mathrm{~V})$, or c. $1968+1 \mathrm{G}>\mathrm{A}(\mathrm{IVS} 11+1 \mathrm{G}>\mathrm{A})$ (Figure 1). ${ }^{9}$ Noteworthy, several patients with atypical progeroid syndromes, which have been referred to as atypical progeroid syndromes (also called atypical HGPS, nonclassical progeria, or atypical WS), do not carry LMNA splicing mutations, but other heterozygous, homozygous, or compound heterozygous mutations in the LMNA gene..$^{24}$ The clinical features of those patients include growth retardation and involve the same body systems (bones, body fat, skin, and hair) as in classical HGPS, but the course and severity of the symptoms vary. The patients have differing ages of onset and symptom severity, with some nearly as severe as 
HGPS, others (such as p.R435C) leading to atypically mild RD, but most are far less severe. ${ }^{24}$ For example, patients affected by atypical WS have early onset of aging phenotypes and an accelerated rate of disease progression than typical WS individuals; they also commonly show absence of bilateral cataracts and diabetes, which are common features in typical WS. ${ }^{25}$ Over 20 LMNA mutations causing atypical progeroid syndromes have been discovered and have been recently reviewed by Doubaj et al, ${ }^{23}$ these are summarized in Figure 1.

In this regard, a dominantly inherited premature aging syndrome that includes prominent cardiovascular and cutaneous manifestations, called LMNA-associated cardiocutaneous progeria syndrome, was recently described. ${ }^{26}$ The disease showed a later onset than classical HGPS, with skin manifestations of aging appearing in the third decade of life. Affected individuals also developed CVD leading to death in their fourth decade. One affected individual in this family also showed primary malignancies not previously reported in $L M N A$-linked progeria disorders. This atypical progeroid syndrome was caused by a novel LMNA mutation (c.899A $>$ G, p.D300G) leading to abnormalities of the nuclear membrane architecture. ${ }^{26}$

There is also indication from studies in nonagenarians suggesting that common LMNA polymorphisms and haplotypes may play a role in the human lifespan. ${ }^{27}$ In this regard, Rodríguez and Eriksson ${ }^{28}$ have recently observed that an $L M N A$ single nucleotide polymorphism (rs4641) results in low and high expressing alleles of the LMNA gene, and might account for the variability in phenotype seen among HGPS individuals. ${ }^{28}$ Noteworthy, not all LMNA mutations cause progeria. More than 450 mutations of the gene have been described causing several different autosomal dominant or recessive diseases collectively called primary laminopathies, and these include muscular dystrophies, lipodystrophies, neuropathies, cardiomyopathies, and the above-discussed segmental progeroid syndromes. ${ }^{26,29}$

\section{Werner syndrome}

WS is a rare autosomal recessive disorder also called adult progeria, and represents the most studied disease model of premature aging in adulthood. ${ }^{30}$ In the Japanese population, a founder effect has been described, and the frequency of WS has been roughly estimated to be $1: 100,000 .{ }^{30}$ Another cluster of WS has been identified in Sardinia, with 18 described cases due to a founder effect. ${ }^{31}$ The prevalence of heterozygous carriers in Japan is approximately $1 / 167$, and it is estimated to be approximately $1 / 120$ in Sardinia. ${ }^{31}$ Outside of Japan the disease prevalence is estimated to be approximately $1: 1,000,000-1: 10,000,000 .^{30}$

The syndrome was first described in the doctoral thesis of Werner $^{32}$ in 1904 . According to a recent report, 1,487 WS cases have been recorded from 1904 to the end of $2008-1,128$ in Japan and 359 outside Japan..$^{33}$ The patients develop features reminiscent of premature aging beginning in the second decade of life, including grey hair, alopecia, prematurely aged face with beaked nose, skin atrophy with scleroderma-like lesions, ischemic heart disease, osteoporosis, bilateral cataracts, type 2 diabetes mellitus, lipodystrophy, and hypogonadism. They also experience an increased risk of cancers, and in most cases, they die because of malignant tumors or arteriosclerosis during the fourth and fifth decades of life. ${ }^{30}$

The disease is caused by mutations of the WRN gene, which encodes the WRN protein, a member of the RecQ DNA helicase family. ${ }^{34} \mathrm{WRN}$ is a multifunctional nuclear protein that maintains genome stability by means of DNA-dependent adenosine triphosphatase (ATPase), , $3^{\prime} \rightarrow 5^{\prime}$ helicase, $3^{\prime} \rightarrow 5^{\prime}$ exonuclease, and DNA strand annealing activities. ${ }^{35}$ Most of the WRN mutations result in the production of truncated proteins lacking the nuclear localization signal, with the subsequent absence of functional WRN protein in nuclei. ${ }^{36}$ WRN has several functional domains and is considered to be a "caretaker of the genome" since it participates in distinct DNA metabolic pathways, including DNA replication, DNA recombination, telomere maintenance, apoptosis, and DNA repair. ${ }^{36}$ Cells isolated from WS individuals display increased chromosomal aberrations and premature senescence in culture, as well as accelerated telomere shortening and several defects in DNA replication. ${ }^{37}$

In $20 \%$ of cases, WS is not caused by $W R N$ gene mutations, but often by mutations in the LMNA gene. ${ }^{25}$ As discussed in the previous section of this paper, WS caused by $L M N A$ mutations is referred to as atypical Werner's syndrome. ${ }^{25}$

\section{WS-associated symptoms}

The lack of a pubertal growth spurt during the teen years is the first clinical sign in WS individuals, leading to a characteristic short stature and low bodyweight. A recent analysis of 196 WS Japanese cases reported that mean height and bodyweight were $158.3 \mathrm{~cm}$ and $45.3 \mathrm{~kg}$ for male patients, and $148.5 \mathrm{~cm}$ and $37.7 \mathrm{~kg}$ for female patients, respectively. ${ }^{38} \mathrm{In}$ their 20s and 30s, WS individuals begin to manifest alopecia, greying hair, and scleroderma-like skin changes, followed by bilateral cataracts, type 2 diabetes mellitus, hypogonadism, skin ulcers, and osteoporosis. ${ }^{1}$ 
A recent epidemiological study of 196 cases revealed that greying or loss of hair, bird-like faces, cataracts, and skin atrophy were present in $93 \%$ to $99 \% .{ }^{38}$ Other common features were clavus or callus, skin ulcers, flat foot, abnormality of the voice, and calcification in the Achilles tendon, observed in $80 \%-90 \%$ of the subjects. ${ }^{38}$ Similarly, a trend analysis in Japanese WS individuals revealed that bird-like faces and a stocky trunk with extremely thin extremities are still a hallmark of the disease, but the body size, which is also still small, has been expanding in recent years, in concert with the growing constitution of the general Japanese population, and some patients exceeded $177 \mathrm{~cm}$ in height and weighed over $70 \mathrm{~kg} .{ }^{33}$

Abnormal glucose and lipid metabolism, hypogonadism, and bone deformity appear by the fourth decade of life in WS subjects..$^{38}$ Impaired glucose tolerance is reported in $15 \%-20 \%$ of WS subjects, diabetes mellitus in $55 \%-70 \%$, and dyslipidemia in $60 \%-85 \%{ }^{38}$ Fertility in WS patients appears to decline soon after sexual maturity, and hypogonadism has been reported in $40 \%$ of both sexes. ${ }^{38}$ Osteoporosis was observed in more than $60 \%$ of WS cases, while osteoarthritis has not been frequently reported in WS. ${ }^{33,38}$

For several years, the clinical diagnosis of WS was based on the presence of four cardinal signs (cataracts, skin changes, short stature, and greying or loss of hair), which are observable in more than $95 \%$ of the cases, as well as on additional signs (osteoporosis, voice change, atherosclerosis, type 2 diabetes mellitus, and so on). For a definite diagnosis, all the cardinal signs (onset over 10 years old) and two additional signs should have been present. In addition, sequencing of the $W R N$ gene could be performed, and the absence of normal WRN protein would be confirmed by Western blot analysis. ${ }^{4,35}$

Following more recent observations, revised diagnostic criteria have been proposed, ${ }^{38}$ including the following cardinal signs and symptoms (onset over 10 years until 40 years of age): (1) progeroid changes of the hair (gray hair, baldness, and so on); (2) cataracts (bilateral); (3) changes of the skin (atrophic skin, tight skin, clavus, callus); (4) soft-tissue calcification (Achilles tendon, and so on); (5) bird-like face; and (6) abnormal voice (high-pitched, squeaky, hoarse). Additional signs include abnormal glucose and/or lipid metabolism, deformation and abnormality of the bone (osteoporosis, and so on), malignant tumors, parental consanguinity, premature atherosclerosis, hypogonadism, short stature, and low bodyweight. The diagnosis is confirmed if all the cardinal signs are present, or if there is a gene mutation in addition to at least three cardinal signs, and suspected if two or more cardinal signs are present, or if 1-2 cardinal signs in addition to other signs, are present. ${ }^{38}$

An unusual spectrum of cancers has been observed in WS subjects who usually die at a mean age of 53-54 years from cancer or arteriosclerosis. ${ }^{30}$ Controversy exists regarding the degree of brain involvement in WS. ${ }^{1}$ A discussion of recent reports of cancer, atherosclerosis, and nervous system disorders in WS will be provided in the following sections.

\section{Atherosclerosis and cancer in WS}

Complications caused by atherosclerosis and cancer represent the major cause of death in WS subjects. Arteriosclerosis obliterans has been observed in more than $20 \%$ of WS subjects, and coronary heart disease in $11 \%-16 \%$ of them. ${ }^{38}$ Less frequent are cerebral hemorrhage and cerebral infarction, observed in $2 \%-5 \%$ of cases. ${ }^{38}$ According to recent trend analyses, atherosclerosis in WS subjects might result from either abnormal lipid metabolism, or from inflammatory mechanisms. ${ }^{33}$

Concerning the cancer incidence in WS individuals, 339 (23\%) out of the 1,487 WS cases described up to the end of 2008 were diagnosed with cancer, ${ }^{33}$ and a nationwide epidemiological study carried out in Japan from 2009 to 2011 revealed that cancer occurred in more than $40 \%$ of the 196 analyzed WS cases. ${ }^{38}$ Cancer in WS individuals often manifests with early age of onset, a high frequency of specific tumor types, including uncommon tumor types and unusual tumor sites, and with the presence of multiple tumors in individual patients. ${ }^{39}$

Lauper et a $\mathrm{l}^{39}$ have recently provided a detailed and quantitatively rigorous view of cancer type and associated type-specific risk in WS. Frequent neoplasms included: thyroid neoplasms (16.1\%), malignant melanoma (13.3\%), meningioma $(10.9 \%)$, soft tissue sarcomas $(10.1 \%)$, hematologic/lymphoid cancers (9.3\%), and osteosarcomas (7.7\%). Other cancers, including nonmelanoma skin cancer, gastrointestinal cancer, ovarian cancer, genitourinary tract cancers, hepatobiliary cancer, head and neck carcinomas, and breast cancer, were observed in $3 \%-5 \%$ of the cases. Less frequent cancers $(1 \%-2 \%)$ were those of the lung and central nervous system, as well as adrenocortical carcinomas. ${ }^{39} \mathrm{~A}$ trend analysis in Japan revealed that the average age of onset of malignancy in WS increased from 37 years old in 1966 to 49 years old in $2008 .^{33}$

\section{Nervous system disorders in WS}

We recently reviewed neurological abnormalities in premature aging disorders, and they are not constant findings 


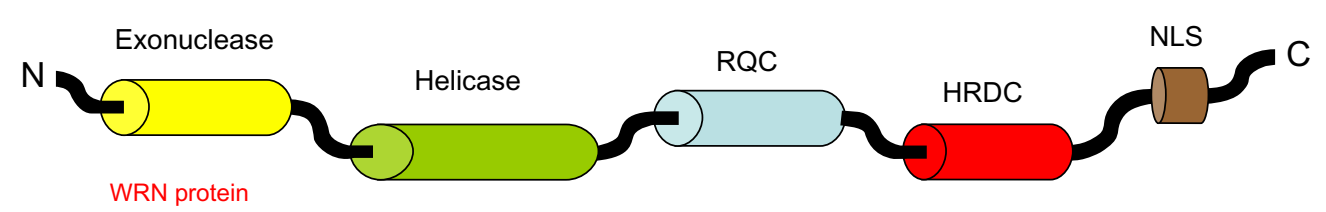

Common WRN gene mutations

Japanese population (founder effect): c.3139-1G>C $\rightarrow$ skipping of exon 26

Sardinian population (founder effect): c.2089-3024A $>\mathrm{G} \rightarrow$ new exon between exons 18 and 19

Dutch population (suspected founder effect): c.3590delA $\rightarrow$ p.N1197fs

Turkish population (suspected founder effect): c.3460-2A>G $\rightarrow$ deletion of exon 30

Moroccan population (suspected founder effect): c.2179dupT $\rightarrow$ p.C727fs

Caucasians (common mutation): c. $1105 \mathrm{C}>\mathrm{T} \rightarrow$ p.R369X: stop codon mutation in exon 9

Figure 2 Representation of the WRN protein and summary of the most common WRN mutations leading to Werner syndrome.

Notes: The diagram illustrates the functional domains of the WRN protein: exonuclease, helicase, RQC, HRDC, and the NLS. Over 70 insertion/deletion mutations, missense and nonsense mutations, as well as splice mutations have been found in the different domains of the WRN protein. Genetic screening is performed as routine diagnostic tests, and the figure shows the most frequent mutations observed according to ethnic group.

Abbreviations: RQC, RecQ C-terminal domain; HRDC, helicase and RNase D C-terminal domain; NLS, nuclear localization signal; N, amino terminal; C, carboxyl terminal.

in WS. ${ }^{1}$ Brain atrophy has been observed in $40 \%$ of WS patients, but only a few cases of dementia or peripheral neuropathy have been reported. A few cases of dementia have been recorded, but the analysis of amyloid beta peptide and hyperphosphorylated tau protein (the hallmarks of Alzheimer's dementia) revealed that WS individuals do not usually appear to be susceptible to Alzheimer's disease. ${ }^{1}$ Myelopathy, demyelinization, and associated central and/ or peripheral neuropathy have been described in at least six patients. ${ }^{40-44}$ Schizophrenia of the paranoia type was also recorded in about $10 \%$ of patients. ${ }^{33}$

\section{Genetics ofWS}

Typical WS is an autosomal recessive genetic disease. Therefore, the parents of a proband are obligate heterozygotes for a disease-causing mutation, and at conception each sibling of an affected individual has a $25 \%$ chance of being affected and a $50 \%$ chance of being an asymptomatic carrier. Classical WS is caused by mutations of the $W R N$ gene on chromosome 8 . The locus spans approximately $250 \mathrm{~kb}$ and consists of 35 exons. ${ }^{34}$

More than $70 W R N$ gene mutations have been found in WS patients; these are mainly nonsense mutations, insertions, and/ or deletions, as well as splice mutations resulting in the production of truncated proteins lacking the nuclear localization signal, with subsequent absence of functional WRN protein in nuclei. ${ }^{36,45}$ The WRN protein has several functional domains (Figure 2), and mutations leading to WS have been observed in all of them. There are founder mutations reported among Japanese patients (c.3139-1G $>$ C, which results in skipping of exon 26) and in Sardinian patients (c.2089-3024A $>$ G, which creates a new exon between exons 18 and 19)..$^{30,31}$ Potential founder mutations have been reported for Dutch (c.3590delA, p.N1197fs), Turkish (c.3460-2A $>$ G, exon 30 deletion), and Moroccan (c.2179dupT, p.C727fs) patients. ${ }^{36}$ The most common mutation in Caucasians is a stop codon mutation in exon 9 (c.1105C $>$ T, p.R369X), accounting for approximately $20 \%$ of the mutations. ${ }^{45}$ Genetic screening combined with Western blot analysis are performed as routine diagnostic tests, and Figure 2 shows the most frequent mutations observed by ethnic group.

\section{Conclusion}

Advancements in our understanding of the genetic and molecular basis of premature aging disorders have led to a better understanding of the onset and progression of the clinical manifestations. They include, for example, the recent discovery of high- and low-expressing LMNA alleles that might account for the different phenotypes observed in HGPS patients, a better characterization of the mutations leading to impaired or completely absent ZMPSTE24 protein activity, or those in the LMNA gene leading to atypical HGPS, overall accounting for a spectrum of disorders ranging from neonatal progeria to atypical WS. ${ }^{20-28}$

In parallel, the advancements in diagnostic examination techniques, and the creation of disease registers or databases allowing large case-control epidemiological studies in both HGPS and WS patients, have led to a better understanding of the epidemiology of disease-associated signs and symptoms. This has led, for example, to a proposed revision of the clinical diagnostic criteria for WS, to the discovery that cerebral infarctions in HGPS are more frequent than expected, and 
to a better understanding of cancer incidence in WS, among others. ${ }^{12,38,39}$

Treatments for HGPS and WS are only available for the symptoms rather than for the disease itself. For example, aggressive treatment of skin ulcers, cholesterol-lowering drugs if the lipid profile is abnormal, control of type 2 diabetes mellitus, surgical treatment of cataracts, and treatment of malignancies in a standard fashion are among the available medicaments in WS, which are often accompanied by dietary/physical regimens to reduce atherosclerosis risk. ${ }^{1}$

Similarly, drugs, dietary changes, and exercise are recommended for HGPS individuals to counteract atherosclerosis risk, body fat reduction, and atrophy of muscles. ${ }^{1}$ Interestingly, the results of the first clinical trial in HGPS revealed that lonafarnib, a farnesyltransferase inhibitor, resulted in better hearing, improved bone structure, and led to gaining additional weight and/or to increased flexibility of the blood vessels. ${ }^{46}$ In this regard, recent trend analyses in Japanese WS patients are extremely important, ${ }^{33}$ as they showed a delayed onset of typical progeroid phenotypes in WS, which may be explained by the environmental changes including lifestyle and medical improvements, thereby offering a potential for interventional trials.

It is clear from that survey that, in recent years, there has been a trend toward an increase in body size in WS patients, as well as a delayed average age at onset of malignancies that follow the rapid improvement and changes in the average lifespan and lifestyle in the general population all over the world. ${ }^{33}$ The future will be to combine genetic, diagnostic, and epidemiological data in order to clarify several of these gene-environment interactions and their relevance to the onset of disease-related symptoms. In addition, as recently shown by some authors studying HGPS patients, ${ }^{28}$ a deeper characterization of the genetics of prematurely aged individuals might help to explain and predict the onset and progression of some of the symptoms, since several of these symptoms might result from the combined presence of different mutations in the same or different genes, thereby opening a window for personalized interventions.

\section{Disclosure}

The author reports no conflicts of interest in this work.

\section{References}

1. Coppedè F. Premature aging syndrome. Adv Exp Med Biol. 2012;724: 317-331.

2. Pollex RL, Hegele RA. Hutchinson-Gilford progeria syndrome. Clin Genet. 2004;66(5):375-381.
3. Hutchinson J. Congenital absence of hair and mammary glands with atrophic condition of the skin and its appendages, in a boy whose mother had been almost wholly bald from alopecia areata from the age of six. Med Chir Trans. 1886;69:473-477.

4. Gilford H. Progeria: a form of senilism. Practitioner. 1904;73:188-217.

5. Eriksson M, Brown WT, Gordon LB, et al. Recurrent de novo point mutations in lamin A cause Hutchinson-Gilford progeria syndrome. Nature. 2003;423(6937):293-298.

6. Dechat T, Pfleghaar K, Sengupta K, et al. Nuclear lamins: major factors in the structural organization and function of the nucleus and chromatin. Genes Dev. 2008;22(7):832-853.

7. Goldman RD, Shumaker DK, Erdos MR, et al. Accumulation of mutant lamin A causes progressive changes in nuclear architecture in Hutchinson-Gilford progeria syndrome. Proc Natl Acad Sci U S A. 2004;101(24):8963-8968

8. Hennekam RC. Hutchinson-Gilford progeria syndrome: review of the phenotype. Am J Med Genet A. 2006;140(23):2603-2624.

9. Gordon LB, Brown WT, Collins FS. Hutchinson-Gilford progeria syndrome. In: Pagon RA, Adam MP, Bird TD, Dolan CR, Fong CT, Stephens K, editors. GeneReviews [Internet]. Seattle, WA: University of Washington, Seattle; 1993-2013. [updated 2011].

10. Qi YC, Xie XH. Hutchinson-Gilford progeria syndrome and its relevance to cardiovascular diseases and normal aging. Biomed Environ Sci. 2013;26(5):382-389.

11. Gerhard-Herman M, Smoot LB, Wake N, et al. Mechanisms of premature vascular aging in children with Hutchinson-Gilford progeria syndrome. Hypertension. 2012;59(1):92-97.

12. Silvera VM, Gordon LB, Orbach DB, Campbell SE, Machan JT, Ullrich NJ. Imaging characteristics of cerebrovascular arteriopathy and stroke in Hutchinson-Gilford progeria syndrome. AJNR Am J Neuroradiol. 2013;34(5):1091-1097.

13. Schmidt E, Nilsson O, Koskela A, et al. Expression of the HutchinsonGilford progeria mutation during osteoblast development results in loss of osteocytes, irregular mineralization, and poor biomechanical properties. J Biol Chem. 2012;287(40):33512-33522.

14. Cleveland RH, Gordon LB, Kleinman ME, et al. A prospective study of radiographic manifestations in Hutchinson-Gilford progeria syndrome. Pediatr Radiol. 2012;42(9):1089-1098.

15. Ullrich NJ, Silvera VM, Campbell SE, Gordon LB. Craniofacial abnormalities in Hutchinson-Gilford progeria syndrome. AJNR Am J Neuroradiol. 2012;33(8):1512-1518.

16. Guardiani E, Zalewski C, Brewer C, et al. Otologic and audiologic manifestations of Hutchinson-Gilford progeria syndrome. Laryngoscope. 2011;121(10):2250-2255.

17. Kalil KA, Fargalley HS. Hypoparathyroidism in an Egyptian child with Hutchinson-Gilford progeria syndrome: a case report. J Med Case Rep. 2012;6:17.

18. Coppedè F, Migliore L. DNA repair in premature aging disorders and neurodegeneration. Curr Aging Sci. 2010;3(1):3-19.

19. De Sandre-Giovannoli A, Bernard R, Cau P, et al. Lamin a truncation in Hutchinson-Gilford progeria. Science. 2003;300(5628):2055.

20. Moulson CL, Fong LG, Gardner JM, et al. Increased progerin expression associated with unusual LMNA mutations causes severe progeroid syndromes. Hum Mutat. 2007;28(9):882-889.

21. Fukuchi K, Katsuya T, Sugimoto K, et al. LMNA mutation in a 45 year old Japanese subject with Hutchinson-Gilford progeria syndrome. J Med Genet. 2004;41(5):e67.

22. Shalev SA, De Sandre-Giovannoli A, Shani AA, Levy N. An association of Hutchinson-Gilford progeria and malignancy. Am J Med Genet A. 2007;143A(16):1821-1826.

23. Doubaj Y, De Sandre-Giovannoli A, Vera EV, et al. An inherited LMNA gene mutation in atypical Progeria syndrome. Am J Med Genet A. 2012;158A(11):2881-2887.

24. Barrowman J, Wiley PA, Hudon-Miller SE, Hrycyna CA, Michaelis S. Human ZMPSTE24 disease mutations: residual proteolytic activity correlates with disease severity. Hum Mol Genet. 2012;21(18): 4084-4093. 
25. Chen L, Lee L, Kudlow BA, et al. LMNA mutations in atypical Werner's syndrome. Lancet. 2003;362(9382):440-445.

26. Kane MS, Lindsay ME, Judge DP, et al. LMNA-associated cardiocutaneous progeria: An inherited autosomal dominant premature aging syndrome with late onset. Am J Med Genet A. 2013;161(7):1599-1611.

27. Conneely KN, Capell BC, Erdos MR, et al. Human longevity and common variations in the LMNA gene: a meta-analysis. Aging Cell. 2012;11(3):475-481.

28. Rodríguez S, Eriksson M. Low and high expressing alleles of the LMNA gene: implications for laminopathy disease development. PLOS ONE. 2011;6(9):e25472.

29. Jacob KN, Garg A. Laminopathies: multisystem dystrophy syndromes. Mol Genet Metab. 2006;87(4):289-302.

30. Goto M. Clinical aspects of Werner's syndrome: its natural history and the genetics of the disease. In: Lebel M, editor. Molecular Mechanisms of Werner's Syndrome. New York, NY: Kluver Academic Plenum Publishers; 2004:1-11.

31. Masala MV, Scapaticci S, Olivieri C, et al. Epidemiology and clinical aspects of Werner's syndrome in North Sardinia: description of a cluster. Eur J Dermatol. 2007;17(3):213-216.

32. Werner O. On Cataract in Conjunction with Scleroderma [dissertation]. Kiel, Germany: Schmidt and Klaunig; 1904.

33. Goto M, Ishikawa Y, Sugimoto M, Furuichi Y. Werner syndrome: a changing pattern of clinical manifestations in Japan (1917 2008). Biosci Trends. 2013;7(1):13-22.

34. Yu CE, Oshima J, Fu YH, et al. Positional cloning of the Werner's syndrome gene. Science. 1996;272(5259):258-262.

35. Muftuoglu M, Oshima J, von Kobbe C, Cheng WH, Leistritz DF, Bohr VA. The clinical characteristics of Werner syndrome: molecular and biochemical diagnosis. Hum Genet. 2008;124(4):369-377.

36. Friedrich K, Lee L, Leistritz DF, et al. WRN mutations in Werner syndrome patients: genomic rearrangements, unusual intronic mutations and ethnic-specific alterations. Hum Genet. 2010;128(1):103-111.
37. Melcher R, von Golitschek R, Steinlein C, et al. Spectral karyotyping of Werner syndrome fibroblast cultures. Cytogenet Cell Genet. 2000;91(1-4):180-185.

38. Takemoto M, Mori S, Kuzuya M, et al. Diagnostic criteria for Werner syndrome based on Japanese nationwide epidemiological survey. Geriatr Gerontol Int. 2013;13(2):475-481.

39. Lauper JM, Krause A, Vaughan TL, Monnat RJ. Spectrum and risk of neoplasia in Werner syndrome: a systematic review. PLOS ONE. 2013;8(4):e59709.

40. Anderson NE, Haas LF. Neurological complications of Werner's syndrome. J Neurol. 2003;250(10):1174-1178.

41. Malandrini A, Dotti MT, Villanova M, Battisti C, Federico A. Neurological involvement in Werner's syndrome: clinical and biopsy study of a familial case. Eur Neurol. 2000;44(3):187-189.

42. Umehara F, Abe M, Nakagawa M, et al. Werner's syndrome associated with spastic paraparesis and peripheral neuropathy. Neurology. 1993;43(6):1252-1254.

43. Just A, Canaple S, Joly H, Piussan C, Rosa A. Complications neurologiques dans un cas de syndrome de Werner [Neurologic complications in a case of Werner syndrome.] Rev Neurol (Paris). 1996;152(10): 634-636. French.

44. Kawamura H, Mori S, Murano S, Yokote K, Tamura K, Saito Y. [Werner's syndrome associated with progressive subcortical vascular encephalopathy of the Binswanger type.] Nihon Ronen Igakkai Zasshi. 1999;36(9):648-651. Japanese.

45. Hisama FM, Kubisch C, Martin GM, Oshima J. Clinical utility gene card for: Werner syndrome. Eur J Hum Genet. 2012;20(5).

46. Gordon LB, Kleinman ME, Miller DT, et al. Clinical trial of a farnesyltransferase inhibitor in children with Hutchinson-Gilford progeria syndrome. Proc Natl Acad Sci U S A. 2012;109(41): 16666-16671.
Clinical Interventions in Aging

\section{Publish your work in this journal}

Clinical Interventions in Aging is an international, peer-reviewed journal focusing on evidence-based reports on the value or lack thereof of treatments intended to prevent or delay the onset of maladaptive correlates of aging in human beings. This journal is indexed on PubMed Central, MedLine, the American Chemical Society's 'Chemical Abstracts Ser-

\section{Dovepress}

vice' (CAS), Scopus and the Elsevier Bibliographic databases. The manuscript management system is completely online and includes a very quick and fair peer-review system, which is all easy to use. Visit http://www.dovepress.com/testimonials.php to read real quotes from published authors. 